J. Korean Math. Soc. 52 (2015), No. 4, pp. 839-851

http://dx.doi.org/10.4134/JKMS.2015.52.4.839

\title{
A NOTE ON STRONGLY *-CLEAN RINGS
}

\author{
JiAn Cui AND Zhou Wang
}

\begin{abstract}
A *-ring $R$ is called (strongly) *-clean if every element of $R$ is the sum of a projection and a unit (which commute with each other). In this note, some properties of $*$-clean rings are considered. In particular, a new class of $*$-clean rings which called strongly $\pi$-*-regular are introduced. It is shown that $R$ is strongly $\pi$-*-regular if and only if $R$ is $\pi$-regular and every idempotent of $R$ is a projection if and only if $R / J(R)$ is strongly regular with $J(R)$ nil, and every idempotent of $R / J(R)$ is lifted to a central projection of $R$. In addition, the stable range conditions of $*$-clean rings are discussed, and equivalent conditions among $*$-rings related to *-cleanness are obtained.
\end{abstract}

\section{Introduction}

Rings in which every element is the product of a unit and an idempotent are said to be unit regular. Recall that an element of a ring $R$ is clean if it is the sum of an idempotent and a unit, and $R$ is clean if every element of $R$ is clean (see [12]). Clean rings were introduced by Nicholson in relation to exchange rings and have been extensively studied since then. Recently, Wang et al. [16] showed that unit regular rings have idempotent stable range one (i.e., whenever $a R+b R=R$ with $a, b \in R$, there exists $e^{2}=e \in R$ such that $a+b e \in U(R)$, written $\operatorname{isr}(R)=1$ for short), and rings with $\operatorname{isr}(R)=1$ are clean. In 1999, Nicholson [13] called an element of a ring $R$ strongly clean if it is the sum of a unit and an idempotent that commute with each other, and $R$ is strongly clean if each of its elements is strongly clean. Clearly, a strongly clean ring is clean, and the converse holds for an abelian ring (that is, all idempotents in the ring are central). Local rings and strongly $\pi$-regular rings are well-known examples of strongly clean rings.

A ring $R$ is a *-ring (or ring with involution) if there exists an operation * $: R \rightarrow R$ such that for all $x, y \in R$

$$
(x+y)^{*}=x^{*}+y^{*}, \quad(x y)^{*}=y^{*} x^{*}, \text { and }\left(x^{*}\right)^{*}=x .
$$

An element $p$ of a $*$-ring is a projection if $p^{2}=p=p^{*}$. Obviously, 0 and 1 are

Received October 6, 2014.

2010 Mathematics Subject Classification. 16W10, 16 U99.

Key words and phrases. (strongly) *-clean ring, (strongly) clean ring, strongly $\pi$-*-regular ring, stable range condition. 
projections of any $*$-ring. A $*$-ring $R$ is $*$-regular [2] if for every $x$ in $R$ there exists a projection $p$ such that $x R=p R$. Following Vaš [15], an element of a *-ring $R$ is (strongly) *-clean if it can be expressed as the sum of a unit and a projection (that commute), and $R$ is (strongly) $*$-clean if all of its elements are (strongly) $*$-clean. Clearly, *-clean rings are clean and strongly $*$-clean rings are strongly clean. It was shown in $[7,11]$ that there exists a clean $*$-ring but not $*$-clean, and unit regular $*$-regular rings (which called $*$-unit regular rings in [7]) need not be strongly $*$-clean, which answered two questions raised by Vaš in $[15]$.

In this note, we continue the study of (strongly) *-clean rings. In Section 2, several basic properties of (strongly) $*$-clean rings are investigated. Motivated by the close relationship between strong $\pi$-regularity and strong cleanness, we introduce the concept of strongly $\pi$-*-regular rings in Section 3 . The structure of strongly $\pi$-*-regular rings is considered and some properties of extensions are discussed. As we know, it is still an open question that whether a strongly clean ring has idempotent stable range one, or even has stable range one (see $[13])$. In Section 4 , we extend $\operatorname{isr}(R)=1$ to the $*$-version. We call a $*$-ring $R$ have projection stable range one (written $\operatorname{psr}(R)=1$ ) if, for any $a, b \in R$, $a R+b R=R$ implies that $a+b p$ is a unit of $R$ for some projection $p \in R$. It is shown that if $R$ is strongly $*$-clean, then $\operatorname{psr}(R)=1$, and if $\operatorname{psr}(R)=1$, then $R$ is $*$-clean. Furthermore, several equivalent conditions among (strongly) clean rings, (strongly) $*$-clean rings and $*$-rings with projection (idempotent) stable range one are obtained.

Throughout this paper, rings are associative with unity. Let $R$ be a ring. The set of all idempotents, all nilpotents and all units of $R$ are denoted by $I d(R), R^{\text {nil }}$ and $U(R)$, respectively. For $a \in R$, the commutant of $a$ is denoted by $\operatorname{comm}(a)=\{x \in R: a x=x a\}$. We write $M_{n}(R)$ for the ring of all $n \times n$ matrices over $R$ whose identity element we write as $I_{n}$. Let $\mathbb{Z}_{n}$ be the ring of integers modulo $n$. For a *-ring $R$, the symbol $P(R)$ stands for the set of all projections of $R$.

\section{2. *-clean rings}

In this section, some basic properties of $*$-clean rings are discussed, and several examples related to $*$-cleanness are given.

Example 2.1. (1) Units, elements in $J(R)$ and nilpotents of a $*$-ring $R$ are *-clean.

(2) Idempotents of a $*$-regular rings are $*$-clean.

Proof. (1) It is obvious.

(2) Let $R$ be $*$-regular and $e \in I d(R)$. Then there exists a projection $p$ such that $(1-e) R=p R$. So we have $1-e=p(1-e)$ and $p=(1-e) p$, and hence $e p=0$. Note that $(e-p)(e-p)=e-e p-p e+p=e+p(1-e)=e+(1-e)=1$. So $e-p \in U(R)$, and $e=p+(e-p)$ is $*$-clean in $R$. 
By Example 2.1, every local ring with involution $*$ is $*$-clean. In [15], Vaš asked whether there is an example of a $*$-ring that is clean but not $*$-clean. It was answered affirmatively in [7] and [11]. In fact, one can construct some counterexamples based on the following.

Example 2.2. Let $R$ be a boolean $*$-ring. Then $R$ is $*$-clean if and only if $*=1_{R}$ is the identity map of $R$. In particular, $R=\mathbb{Z}_{2} \oplus \mathbb{Z}_{2}$ with $(a, b)^{*}=(b, a)$ is clean but not $*$-clean.

Proof. Note that every boolean ring is clean. Suppose that $R$ is $*$-clean. Given any $a \in R$. Then $-a=p+u=p+1=p-1$ for some $p \in P(R)$. So we have $a=1-p \in P(R)$. Thus, $a^{*}=a$, which implies $*=1_{R}$. Conversely, if $*=1_{R}$, then every idempotent of $R$ is a projection. Thus, $R$ is $*$-clean.

Lemma 2.3. Let $R$ be $a$ *-ring. If $2 \in U(R)$, then for any $u^{2}=1, u^{*}=u \in R$ if and only if every idempotent of $R$ is a projection.

Proof. $(\Rightarrow)$ Let $e \in \operatorname{Id}(R)$. Then $(1-2 e)^{2}=1$. So we have $2 e=2 e^{*}$, and thus $2\left(e-e^{*}\right)=0$. Since $2 \in U(R), e=e^{*}$. As desired.

$(\Leftarrow)$ Given $u \in R$ with $u^{2}=1$. Then $\frac{u+1}{2} \in I d(R)$ since $\left(\frac{u+1}{2}\right)^{2}=\frac{u^{2}+2 u+1}{4}=$ $\frac{u+1}{2}$. Since every idempotent of $R$ is a projection, it follows from $\left(\frac{u+1}{2}\right)^{*}=\frac{u+1}{2}$ that $u^{*}=u$.

The *-ring $R=\mathbb{Z}_{2} \oplus \mathbb{Z}_{2}$ in Example 2.2 reveals that " $2 \in U(R)$ " in Lemma 2.3 cannot be removed.

Corollary 2.4. Let $R$ be a *-ring with $2 \in U(R)$. The following are equivalent:

(1) $R$ is clean and every unit of $R$ is self-adjoint (i.e., $u^{*}=u$ for every $u \in U(R))$.

(2) $R$ is $*$-clean and $*=1_{R}$.

Proof. $(2) \Rightarrow(1)$ is trivial.

$(1) \Rightarrow(2)$. Let $a \in R$. Then $a=e+u$ for some $e \in I d(R)$ and $u \in U(R)$. Note that $(1-2 e)^{2}=1$. By Lemma $2.3, e^{*}=e$. Thus $a \in R$ is $*$-clean and $a^{*}=a$, and so $*=1_{R}$.

Recall that an element $t$ of a $*$-ring $R$ is self-adjoint square root of 1 if $t^{2}=1$ and $t^{*}=t$.

Theorem 2.5. Let $R$ be a *-ring, the following are equivalent:

(1) $R$ is *-clean and $2 \in U(R)$.

(2) Every element of $R$ is a sum of a unit and a self-adjoint square root of 1.

Proof. (1) $\Rightarrow(2)$. Let $a \in R$. Then $\frac{1+a}{2}=p+u$ for some $p \in P(R)$ and $u \in U(R)$. It follows that $a=(2 p-1)+2 u$ where $(2 p-1)^{*}=2 p-1,(2 p-1)^{2}=1$ and $2 u \in U(R)$.

$(2) \Rightarrow(1)$. We first show that $2 \in U(R)$. By hypothesis, $1=x+v$ with $x^{2}=1$ and $v \in U(R)$. So we have $(1-v)^{2}=x^{2}=1$, which implies that 
$v^{2}=2 v$. Since $v$ is a unit, $v=2 \in U(R)$. Given any $a \in R$, then there exist $y, w \in R$ satisfying $2 a-1=y+w$ with $y^{*}=y, y^{2}=1$ and $w \in U(R)$. Thus, $a=\frac{y+1}{2}+\frac{w}{2}$ is a $*$-clean expression since $\left(\frac{y+1}{2}\right)^{*}=\frac{y+1}{2},\left(\frac{y+1}{2}\right)^{2}=\frac{y+1}{2}$ and $\frac{w}{2} \in U^{2}(R)$.

Camillo and $\mathrm{Yu}$ [5] showed that if $R$ is a ring in which 2 is a unit, then $R$ is clean if and only if every element of $R$ is the sum of a unit and a square root of 1 . Indeed, by the proof of Theorem 2.5, the condition $2 \in U(R)$ is also necessary.

Proposition 2.6. The following are equivalent for a $*$-ring $R$ :

(1) $R$ is *-clean and 0,1 are the only projections.

(2) $R$ is clean ring and 0,1 are the only idempotents.

(3) $R$ is a local ring.

Proof. $(2) \Rightarrow(3)$ follows from [14, Lemma 14] and $(3) \Rightarrow(1)$ follows by Example 2.1.

$(1) \Rightarrow(2)$. It suffices to show that the only idempotents in $R$ are 0 and 1 . For $e^{2}=e \in R$, the hypothesis implies that $e=p+u$ where $p \in P(R)=\{0,1\}$ and $u \in U(R)$. If $p=0$, then $e=u$ is a unit, so $e=1$. If $p=1$, then $1-e=-u \in U(R)$, and hence $e=0$. As required.

Let $I$ be an ideal of a $*$-ring $R$. We call $I$ is $*$-invariant if $I^{*} \subseteq I$. In this case, the involution $*$ of $R$ can be extended to the factor $\operatorname{ring} R / I$, which is still denoted by $*$.

Lemma 2.7. Let $R$ be *-clean. If $I$ is a*-invariant ideal of $R$, then $R / I$ is *-clean. In particular, $R / J(R)$ is *-clean.

Proof. Since the homomorphism image of a projection (resp., unit) is also a projection (resp., unit), the result follows.

Next we only need to prove that $J(R)$ is $*$-invariant. For any $a^{*} \in(J(R))^{*}$, we show that $a^{*} \in J(R)$. Note that $a \in J(R)$. Take any $x \in R$. Then $1-x^{*} a \in$ $U(R)$. Thus $1-a^{*} x=\left(1-x^{*} a\right)^{*}$ is a unit of $R$, as desired.

Let $R$ be a $*$-ring. Then $*$ induces an involution of the power series ring $R[[x]]$, denoted by $*$, where $\left(\sum_{i=0}^{\infty} a_{i} x^{i}\right)^{*}=\sum_{i=0}^{\infty} a_{i}^{*} x^{i}$.

Proposition 2.8. Let $R$ be $a *$-ring. Then $R[[x]]$ is *-clean if and only if $R$ is $*$-clean.

Proof. Suppose that $R[[x]]$ is $*$-clean. Note that $R \cong R[[x]] /(x)$ and $(x)$ is a *invariant ideal of $R[[x]]$. By Lemma 2.7, $R$ is $*$-clean. Conversely, assume that $R$ is $*$-clean. Let $f(x)=\sum_{i=0}^{\infty} a_{i} x^{i} \in R[[x]]$. Write $a_{0}=p+u$ with $p \in P(R)$ and $u \in U(R)$. Then $f(x)=p+\left(u+\sum_{i=1}^{\infty} a_{i} x^{i}\right)$, where $p \in P(R) \subseteq P(R[[x]])$ and $u+\sum_{i=1}^{\infty} a_{i} x^{i} \in U(R[[x]])$. Hence $f(x)$ is $*$-clean in $R[[x]]$.

According to [14, Proposition 13], the polynomial ring $R[x]$ is never clean. Hence, $R[x]$ is not $*$-clean for any involution $*$. 


\section{Strongly $\pi-*$-regular rings}

Strong $\pi$-regularity is closely related to strong cleanness. In this section, we introduce the notion of strongly $\pi-*$-regular rings which can be viewed as $*$-versions of strongly $\pi$-regular rings. The structure and properties of strongly $\pi$-*-regular rings are given.

Lemma 3.1 ([11, Lemma 2.1]). Let $R$ be a $*$-ring. If every idempotent of $R$ is a projection, then $R$ is abelian.

Due to [7], an element $a$ of a $*$-ring $R$ is strongly $*$-regular if $a=p u=u p$ with $p \in P(R)$ and $u \in U(R) ; R$ is strongly $*$-regular if each of its elements is strongly $*$-regular. By $[7$, Proposition 2.8], any strongly $*$-regular element is strongly $*$-clean.

Theorem 3.2. Let $R$ be $a *$-ring. Then the following are equivalent for $a \in R$ :

(1) There exist $e \in P(R), u \in U(R)$ and an integer $m \geq 1$ such that $a^{m}=e u$ and $a, e, u$ commute with each other.

(2) There exist $f \in P(R), v \in U(R)$ such that $a=f+v, f v=v f$ and af $\in R^{\text {nil }}$.

(3) There exists $p \in P(R)$ such that $p \in \operatorname{comm}(a)$, ap $\in U(p R p)$ and $a(1-p) \in R^{\text {nil }}$.

(4) There exists $b \in \operatorname{comm}(a)$ such that $(a b)^{*}=a b, b=b a b$ and $a-a^{2} b \in$ $R^{\text {nil }}$.

Proof. (1) $\Rightarrow(2)$. Write $f=1-e$. Clearly, $f \in P(R)$ and $a^{m}-f \in U(R)$ with the inverse $u^{-1} e-f$. From $a f=f a$, we have $a-f:=v$ is a unit of $R$ (since $\left.(a-f)\left(a^{m-1}+a^{m-2} f+\cdots+a f+f\right)=a^{m}-f \in U(R)\right)$ and $f v=v f$. It is clear that $(\text { af })^{m}=a^{m} f=0$.

$(2) \Rightarrow(3)$. Set $p=1-f$. Then $p \in P(R), a p=p a=v p \in U(p R p)$ and $a(1-p)=a f \in R^{\text {nil }}$.

$(3) \Rightarrow(4)$. By (3), $a w=w a=p$ for some $w \in U(p R p)$. So we obtain $[a-(1-p)][w-(1-p)]=1-a(1-p) \in U(R)$ since $a(1-p)$ is nilpotent, which implies that $a-(1-p) \in U(R)$. Let $b=[a-(1-p)]^{-1} p$. Then $b \in \operatorname{comm}(a)$, $b p=b$ and $a b=[a-(1-p)] b=p \in P(R)$. Thus $(a b)^{*}=a b, b=b p=b a b$ and $a-a^{2} b=a(1-a b)=a(1-p) \in R^{\mathrm{nil}}$.

$(4) \Rightarrow(1)$. Let $e=a b$. Then $(a b)^{*}=a b$ implies $e^{*}=e$, and $b a b=b$ yields $e^{2}=e$. So $e \in P(R)$. As $a-a^{2} b \in R^{\text {nil }}, a^{m}=a^{m} e$ for some integer $m \geq 1$. Take $u=a^{m}+(1-e)$ and $u^{\prime}=b^{m} e+(1-e)$. Then $u u^{\prime}=u^{\prime} u=1$. Hence, $u \in U(R)$ and $a^{m}=a^{m} e=u e$ with $a, e, u$ commuting with each other.

Recall that an element $a$ of a ring $R$ is strongly $\pi$-regular if $a^{n} \in a^{n+1} R \cap$ $R a^{n+1}$ for some $n \geq 1$ (equivalently, $a^{n}=e u$ with $e \in I d(R), u \in U(R)$ and $a, e, u$ all commute [13]); $R$ is strongly $\pi$-regular if every element of $R$ is strongly $\pi$-regular. Based on the above, we introduce the following concept. 
Definition 3.3. Let $R$ be a *-ring. An element $a \in R$ is called strongly $\pi-*-$ regular if it satisfies the conditions in Theorem $3.2 ; R$ is called strongly $\pi$-*-regular if every element of $R$ is strongly $\pi$-*-regular.

Corollary 3.4. Any strongly *-regular element is strongly $\pi$-*-regular, and any strongly $\pi$-*-regular element is strongly $*$-clean.

Example 3.5. (1) Let $R=\mathbb{Z}_{4}$ and $*=1_{R}$. Then $R$ is strongly $\pi$-*-regular. However, $2 \in R$ is not strongly $*$-regular.

(2) Let $R$ be a local domain with involution $*$ and $J(R) \neq 0$. Note that $P(R)=I d(R)=\{0,1\}$. So $R$ is strongly $*$-clean by Proposition 2.6 , but any power of a nonzero element in $J(R)$ can not expressed as the product of a projection and a unit.

Recall that a ring $R$ is $\pi$-regular if for any $a \in R$, there exist $n \geq 1$ and $b \in R$ such that $a^{n}=a^{n} b a^{n}$. Strongly $\pi$-regular rings and regular rings are $\pi$-regular (see [13]). A ring $R$ is directly finite if $a b=1$ implies $b a=1$ for all $a, b \in R$. Abelian rings are directly finite.

Theorem 3.6. The following are equivalent for $a *$-ring $R$ :

(1) $R$ is strongly $\pi$-*-regular.

(2) $R$ is $\pi$-regular and every idempotent of $R$ is a projection.

(3) For any $a \in R$, there exist $n \geq 1$ and $p \in P(R)$ such that $a^{n} R=p R$, and $R$ is abelian.

(4) For any $a \in R$, there exists $n \geq 1$ such that $a^{n}$ is strongly $*$-regular.

(5) For any $a \in R$, there exist $p \in P(R)$ and $u \in U(R)$ such that $a=p+u$, ap $\in R^{\text {nil }}$; and $v^{-1} q v$ is a projection for all $v \in U(R)$ and all $q \in P(R)$.

Proof. $(1) \Rightarrow(2)$. Note that every strongly $\pi$-*-regular ring is strongly $\pi$ regular and strongly $*$-clean. Thus $R$ is a $\pi$-regular ring. By [11, Theorem 2.2], every idempotent of $R$ is a projection.

$(2) \Rightarrow(3)$. For any $a \in R$, there exists $n \geq 1$ such that $a^{n}=a^{n} x a^{n}$ for some $x \in R$. Write $a^{n} x=p$. Then $p \in P(R)$ and $a^{n}=p a^{n}$. It is clear that $a^{n} R=p R$. In view of Lemma $3.1, R$ is abelian.

$(3) \Rightarrow(4)$. Let $e \in I d(R)$. Then $e R=p R$ for some $p \in P(R)$. Since $R$ is abelian, we have $e=p e=e p=p$. Thus, every idempotent of $R$ is a projection. Given $a \in R$, there exist $n \geq 1$ and $q \in P(R)$ such that $a^{n} R=q R$. So one gets $a^{n}=q a^{n}$ and $q=a^{n} x$ for some $x \in R$, which implies $a^{n}=a^{n} x a^{n}$. Next we show that $a^{n}-(1-q)$ is invertible. Note that $\left[a^{n}-(1-q)\right][x q-(1-q)]=1$. Then $a^{n}-(1-q):=u \in U(R)$ since $R$ is directly finite. Multiplying the equation $a^{n}-(1-q)=u$ by $p$ yields $a^{n}=a^{n} q=u q=q u$, which implies that $a^{n}$ is strongly $*$-regular.

$(4) \Rightarrow(5)$. For $e \in I d(R), e=q v=v q$ for some $q \in P(R)$ and $v \in U(R)$ by the assumption. Then $e=q v=e^{2}=q v^{2}$, and so we obtain $q=q v=e$, which implies that every idempotent of $R$ is a projection. Clearly, $v^{-1} q v$ is a projection for all $v \in U(R)$ and all $q \in P(R)$. Given $a \in R$ as in (4), $a^{n}=$ 
$(1-p) w=w(1-p)$ for some $p \in P(R)$ and $w \in U(R)$. Note that $R$ is abelian. So we have $a^{n} p=(a p)^{n}=0$ and $(a-p)\left[a^{n-1} w^{-1}(1-p)-\sum_{i=0}^{n} a^{i} p\right]=1$, and hence $a-p \in U(R)$ as $R$ is directly finite.

$(5) \Rightarrow(1)$. By $(5)$, every element of $R$ is $*$-clean. In view of $[11$, Theorem $2.2], R$ is abelian. Thus $R$ is strongly $\pi$-*-regular by Theorem $3.2(2)$.

Corollary 3.7. Let $R$ be $a *$-ring. The following are equivalent:

(1) $R$ is strongly $\pi$-*-regular.

(2) $R / J(R)$ is strongly $\pi$-*-regular with $J(R)$ nil, every projection of $R$ is central and every projection of $R / J(R)$ is lifted to a projection of $R$.

(3) $R / J(R)$ is strongly *-regular with $J(R)$ nil, and every idempotent of $R / J(R)$ is lifted to a central projection of $R$.

Proof. Write $\bar{R}=R / J(R)$. By Lemma $2.7, \bar{R}$ is a $*$-ring.

$(1) \Rightarrow(2)$. Clearly, $\bar{R}$ is strongly $\pi$-*-regular. As $R$ is strongly $\pi$-regular, for any $a \in J(R)$, there exist $m \geq 1, e \in I d(R)$ and $u \in U(R)$ such that $e=a^{m} u \in J(R)$. So $a^{m}=e u^{-1}=0$, which implies that $J(R)$ is nil. Note that $R$ is strongly $*$-clean. So the rest follows from [11, Corollary 2.11].

$(2) \Rightarrow(3)$. By virtue of $\left[11\right.$, Corollar 2.11], $\bar{R}$ is reduced (i.e., $\bar{R}^{\text {nil }}=0$ ), and every idempotent of $\bar{R}$ is lifted to a central projection of $R$. So we only need to prove that $\bar{R}$ is strongly $*$-regular. Given any $x \in \bar{R}$. By Theorem 3.2, there exist $p \in P(\bar{R})$ and $v \in U(\bar{R})$ such that $a=p+v, v p=p v$ and $a p \in \bar{R}^{\text {nil }}=0$. It follows that $a=a(1-p)=v(1-p)=(1-p) v$ is strongly $*$-regular in $\bar{R}$.

$(3) \Rightarrow(1)$. Since $\bar{R}$ is strongly regular, it is reduced clean. By $[11$, Corollary 2.11 , every idempotent of $R$ is a projection. Note that $J(R)$ is nil and $\bar{R}$ is $\pi$-regular. So $R$ is $\pi$-regular by [1, Theorem 4]. In view of Theorem 3.6, $R$ is strongly $\pi$-*-regular.

Corollary 3.8. Let $R$ be $a *$-ring. Then $R$ is strongly *-clean and $\pi$-regular if and only if $R$ is strongly $\pi$-*-regular.

Proof. If $R$ is strongly $*$-clean and $\pi$-regular, by [11, Theorem 2.2], idempotents of $R$ are projections. So $R$ is strongly $\pi$-*-regular by Theorem 3.6. The other direction is clear.

For a $*$-ring $R$, the matrix ring $M_{n}(R)$ has a natural involution inherited from $R$ : if $A=\left(a_{i j}\right) \in M_{n}(R), A^{*}$ is the transpose of $\left(a_{i j}^{*}\right)$ (i.e., $A^{*}=\left(a_{i j}^{*}\right)^{T}=$ $\left.\left(a_{j i}^{*}\right)\right)$. Henceforth we consider $M_{n}(R)$ as a $*$-ring with respect to this natural involution.

Corollary 3.9. Let $R$ be a *-ring. Then $M_{n}(R)$ is not strongly $\pi$-*-regular for any $n \geq 2$.

Let $R$ be a $*$-ring and $S=p R p$ with $p \in P(R)$. Then the restriction of $*$ on $S$ will be an involution of $S$, which is also denoted by $*$.

Corollary 3.10. If $R$ is strongly $\pi$-*-regular, then so is eRe for any $e \in \operatorname{Id}(R)$. 
Proof. Let $S=e R e$ with $e \in I d(R)$. By hypothesis, $e$ is a projection of $R$. So $S$ is a $*$-ring. It is well known that $S$ is strongly $\pi$-regular (see also [4, Lemma 39]). Clearly, every idempotent of $S(\subseteq R)$ is a projection. So the result follows by Theorem 3.6.

Let $R G$ be the group ring of a group $G$ over a ring $R$. According to [11, Lemma 2.12], the map * $R G \rightarrow R G$ given by $\left(\sum_{g} a_{g} g\right)^{*}=\sum_{g} a_{g}^{*} g^{-1}$ is an involution of $R G$, and is denoted by $*$ again.

Corollary 3.11. Let $R$ be $a *$-ring with artinian prime factors, $2 \in J(R)$ and $G$ be a locally finite 2-group. Then $R$ is strongly $\pi$-*-regular if and only if $R G$ is strongly $\pi$-*-regular.

Proof. Assume that $R$ is strongly $\pi$-*-regular. Then $\operatorname{Id}(R)=P(R)$. In particular, $R$ is abelian. So idempotents of $R$ coincide with idempotents in $R G$ by [8, Lemma 11], and hence every idempotent of $R G$ is a projection. Since $R$ is a ring with artinian prime factors and $G$ is a locally finite 2-group, $R G$ is a strongly $\pi$-regular ring by [10, Theorem 3.3]. In view of Theorem 3.6, $R G$ is strongly $\pi$-*-regular.

Conversely, $R$ is strongly $\pi$-regular by [10, Proposition 3.4]. Note that $I d(R) \subseteq I d(R G)$ and all idempotents of $R G$ are projections. By Theorem $3.6, R$ is strongly $\pi-*$-regular.

Let $\mathbb{C}$ be the complex filed. It is well known that for any $n \geq 1$, the matrix ring $M_{n}(\mathbb{C})$ is strongly $\pi$-regular. However, $M_{n}(\mathbb{C})$ is not strongly $\pi$-*-regular whenever $n \geq 2$ by Corollary 3.9. So it is interesting to determine when a matrix of $M_{n}(\mathbb{C})$ is strongly $\pi$-*-regular. The set of all $n \times 1$ matrices over $\mathbb{C}$ is denoted by $\mathbb{C}^{n}$.

Example 3.12. Let $S=M_{n}(\mathbb{C})$ with $*$ the transpose operation. Then $A$ is strongly $\pi$-*-regular if and only if there exist $e_{1}, e_{2}, \ldots, e_{n} \in \mathbb{C}^{n}$ such that $e_{i}^{*} e_{j}=0$ for $i=1, \ldots, r ; j=r+1, \ldots, n$, and $A=P\left(\begin{array}{cc}C & 0 \\ 0 & N\end{array}\right) P^{-1}$ with $P=$ $\left(e_{1}, e_{2}, \ldots, e_{n}\right) \in U(S), C \in U\left(M_{r}(\mathbb{C})\right)$ and $N \in\left[M_{n-r}(\mathbb{C})\right]^{\text {nil }}$. In particular, any real symmetric matrix is strongly $\pi-*$-regular.

Proof. Given $A \in S$. Assume that $\operatorname{rank}(A)=r$. By the Jordan canonical decomposition, there exists $P=\left(e_{1}, e_{2}, \ldots, e_{n}\right) \in U(S)$ such that

$$
A=P\left(\begin{array}{cc}
C & 0 \\
0 & N
\end{array}\right) P^{-1}
$$

where $e_{i} \in \mathbb{C}^{n}$ for all $i, C \in U\left(M_{r}(\mathbb{C})\right)$ and $N \in\left[M_{n-r}(\mathbb{C})\right]^{\text {nil }}$. Write

$$
B=P\left(\begin{array}{cc}
C^{-1} & 0 \\
0 & 0
\end{array}\right) P^{-1} \text {. }
$$

Then one easily gets that $B A=A B, B=B A B$ and $A-A^{2} B=P\left(\begin{array}{ll}0 & 0 \\ 0 & N\end{array}\right) P^{-1}$ is nilpotent. Note that $B$ satisfies the above conditions is unique (see [3]). In 
view of Theorem 3.2, $A$ is strongly $\pi$-*-regular if and only if $(A B)^{*}=A B$. Notice that $A B=P\left(\begin{array}{cc}I_{r} & 0 \\ 0 & 0\end{array}\right) P^{-1}$ and

$$
\begin{aligned}
& (A B)^{*}=A B \\
\Leftrightarrow & \left(P^{-1}\right)^{*}\left(\begin{array}{rr}
I_{r} & 0 \\
0 & 0
\end{array}\right) P^{*}=P\left(\begin{array}{rr}
I_{r} & 0 \\
0 & 0
\end{array}\right) P^{-1} \\
\Leftrightarrow & \left(P^{*} P\right)^{-1}\left(\begin{array}{rr}
I_{r} & 0 \\
0 & 0
\end{array}\right)\left(P^{*} P\right)=\left(\begin{array}{cc}
I_{r} & 0 \\
0 & 0
\end{array}\right) \\
\Leftrightarrow & \left(\begin{array}{cc}
I_{r} & 0 \\
0 & 0
\end{array}\right)\left(P^{*} P\right)=\left(P^{*} P\right)\left(\begin{array}{cc}
I_{r} & 0 \\
0 & 0
\end{array}\right) \\
\Leftrightarrow & P^{*} P=\left(\begin{array}{cc}
V_{1} & 0 \\
0 & V_{2}
\end{array}\right) \text { with } V_{1} \in U\left(M_{r}(\mathbb{C})\right) \text { and } V_{2} \in U\left(M_{n-r}(\mathbb{C})\right) \\
\Leftrightarrow & e_{i}^{*} e_{j}=0 \text { for all } i \in\{1,2, \ldots, r\}, j \in\{r+1, r+2, \ldots, n\},
\end{aligned}
$$

where

$$
\begin{aligned}
& V_{1}=\left(e_{1}^{*}, e_{2}^{*}, \ldots, e_{r}^{*}\right)^{T}\left(e_{1}, e_{2}, \ldots, e_{r}\right) \\
& V_{2}=\left(e_{r+1}^{*}, e_{r+2}^{*}, \ldots, e_{n}^{*}\right)^{T}\left(e_{r+1}, e_{r+2}, \ldots, e_{n}\right) .
\end{aligned}
$$

If $A \in S$ is a real symmetric matrix, then there exists an orthogonal matrix $P$ (i.e., $\left.P^{-1}=P^{T}=P^{*}\right)$ such that $A=P\left(\begin{array}{rr}I_{r} & 0 \\ 0 & 0\end{array}\right) P^{-1}$. So the result follows.

In view of [2, Proposition 3], the involution of a $*$-regular ring $R$ is proper (i.e., $x^{*} x=0$ implies that $x=0$ for all $x \in R$ ).

Remark 3.13. If $R$ is strongly $\pi$-*-regular, then for any $x \in R, x^{*} x=0$ implies $x \in R^{\text {nil }}$. Indeed, by Theorem 3.2, there exist $p \in P(R)$ and $u \in U(R)$ such that $x^{m}=p u=u p$ for some $m \geq 1$. Then $0=\left(x^{*}\right)^{m} x^{m}=\left(x^{m}\right)^{*} x^{m}=u^{*} p u$, and thus $p=0$, whence $x^{m}=0$.

\section{Stable range conditions}

In [13], Nicholson asked whether every strongly clean ring has stable range one, and it is still open. Recall that a $\operatorname{ring} R$ is said to have idempotent stable range one (written isr $(R)=1$ ) provided that for any $a, b \in R, a R+b R=R$ implies that $a+b e \in U(R)$ for some $e \in \operatorname{Id}(R)$ (see [6, 16]). If $e$ is an arbitrary element of $R$ (not necessary an idempotent), then $R$ is said to have stable range one. Clearly, if $\operatorname{isr}(R)=1$, then $R$ is clean and has stable range one. We extend the notion of $\operatorname{isr}(R)=1$ to $*$-versions.

Definition 4.1. A $*$-ring $R$ is said to have projection stable range one (written $\operatorname{psr}(R)=1)$ if for any $a, b \in R, a R+b R=R$ implies there exists $p \in P(R)$ such that $a+b p \in U(R)$.

The following result is motivated by [6, Proposition 2].

Proposition 4.2. Let $R$ be $a *$-ring. The following are equivalent:

(1) $\operatorname{psr}(R)=1$.

(2) For any $a, b \in R, a R+b R=R$ implies there exists $p \in P(R)$ such that $a+b p$ is right invertible. 
(3) For any $a, b \in R, a R+b R=R$ implies there exists $p \in P(R)$ such that $a+b p$ is left invertible.

Proof. The proof is similar to that of [6, Proposition 2].

$(1) \Rightarrow(2)$ is clear.

$(2) \Rightarrow(3)$. Let $a, b \in R$ with $a R+b R=R$. Then there is a projection $p \in R$ such that $a+b p=u$ is right invertible. Assume that $u w=1$ for some $w \in R$. Then $w R+(1-w u) R=R$. So the hypothesis implies there exists $q \in P(R)$ such that $w+(1-w u) q$ is right invertible. Note that $u[w+(1-w u) q]=1$. Thus $w+(1-w u) q$ is also left invertible, and hence invertible. This implies that $u \in U(R)$.

$(3) \Rightarrow(1)$. Given any $a, b \in R$ with $a R+b R=R$. Then there exists $p \in P(R)$ such that $a+b p$ is left invertible. We may let $v \in R$ with $v(a+b p)=1$. Then $v R+0 R=R$. By hypothesis, we can find a projection $q$ such that $v+0 q=v$ is left invertible. So $v$ is a unit, which implies that $a+b p \in U(R)$. Therefore, $\operatorname{psr}(R)=1$.

For a $*$-ring $R$, it is clear that if $\operatorname{psr}(R)=1$, then $\operatorname{isr}(R)=1$. However, there exists a $*$-ring with $\operatorname{isr}(R)=1$ but not satisfies $\operatorname{psr}(R)=1$.

Example 4.3. Define the involution of $\mathbb{Z}_{2}$ by $*: x \mapsto x$. Let $S=M_{2}\left(\mathbb{Z}_{2}\right)$. Then $S$ is a $*$-ring. In view of [16, Corollary 3.4], $\operatorname{isr}(S)=1$ since $S$ is unit regular. Notice that $P(S)=\left\{O, I_{2},\left(\begin{array}{ll}1 & 0 \\ 0 & 0\end{array}\right),\left(\begin{array}{ll}0 & 0 \\ 0 & 1\end{array}\right)\right\}$, and $\left(\begin{array}{ll}1 & 0 \\ 0 & 0\end{array}\right) S+\left(\begin{array}{ll}0 & 0 \\ 1 & 0\end{array}\right) S=S$. However, $\left(\begin{array}{ll}1 & 0 \\ 0 & 0\end{array}\right)+\left(\begin{array}{ll}0 & 0 \\ 1 & 0\end{array}\right) P$ is not invertible for any $P \in P(S)$. Hence, $\operatorname{psr}(S) \neq 1$.

From Example 4.3, one can also find that the projection stable range one property cannot be inherited to the matrix ring.

Proposition 4.4. Let $R$ be a*-ring. If $\operatorname{psr}(R)=1$, then $R$ is *-clean.

Proof. For any $a \in R$, the equation $a R+(-1) R=R$ implies that $a+(-1) p=$ $u \in U(R)$ for some $p \in P(R)$. So $a=p+u$, and hence $R$ is $*$-clean.

According to [15, Proposition 4], the ring in Example 4.3 is $*$-clean. So we conclude that the converse of Proposition 4.4 is not true.

Following Nicholson [12], a ring $R$ is exchange if for every $a \in R$, there exists $e^{2}=e \in a R$ such that $1-e \in(1-a) R$. Clean rings are exchange, the converse holds whenever the rings are abelian. A *-ring $R$ is called *-abelian if every projection of $R$ is central [15].

Theorem 4.5. Let $R$ be a *-ring. The following are equivalent:

(1) $\operatorname{psr}(R)=1$ and $R$ is *-abelian.

(2) For any $a, b \in R, a R+b R=R$ implies there exists a projection $p \in \operatorname{comm}(a)$ such that $a+b p \in U(R)$.

(3) $\operatorname{isr}(R)=1$ and every idempotent of $R$ is a projection.

(4) $R$ is clean (or exchange) and every idempotent of $R$ is a projection.

(5) $R$ is *-clean and *-abelian.

(6) $R$ is strongly $*$-clean. 
(7) For every $a \in R$, there exists a projection $p \in a R$ such that $1-p \in$ $(1-a) R$.

Proof. (1) $\Rightarrow(2)$ and (3) $\Rightarrow(4)$ are clear; $(4) \Rightarrow(5) \Rightarrow(6) \Rightarrow(7)$ follows from [11, Theorem 2.2].

$(2) \Rightarrow(3)$. We only need to show that all idempotents are projections. Let $e \in I d(R)$. Then $e R+(-1) R=R$. So there exists $p \in P(R)$ such that $e p=p e$ and $e-p \in U(R)$. Note that $(e-p)(1-e-p)=(1-e-p)(e-p)=0$. Thus, $e=1-p \in P(R)$. Therefore, every idempotent of $R$ is a projection.

$(7) \Rightarrow(1)$. Let $e \in I d(R)$. Then there exists a projection $p \in e R$ such that $1-p \in(1-e) R$. So we obtain $p=e p$ and $1-p=(1-e)(1-p)$. It follows that $e=p$, and thus $\operatorname{Id}(R)=P(R)$. In view of Lemma 3.1, $R$ is abelian. Note that $R$ is exchange. Then by [6, Theorem 12], $\operatorname{isr}(R)=1$, and hence $\operatorname{psr}(R)=1$.

It is still unknown that whether strongly clean rings have stable range one ([13]). However, we have an affirmative answer of their $*$-versions.

Corollary 4.6. If $R$ is a strongly $*$-clean ring, then $\operatorname{psr}(R)=1$.

The following example will reveal that the converse of Corollary 4.6 does not hold.

Example 4.7. Let $S=M_{2}\left(\mathbb{Z}_{3}\right)$. The involution of $S$ is defined by $A \rightarrow A^{*}$, where $A^{*}$ is the transpose of $A \in S$. Then $S$ is not strongly *-clean by [7, Theorem 2.3]. Since $S$ is unit regular, $\operatorname{isr}(S)=1$ by [16, Corollary 3.4]. In view of [9, Lemma 7], we have

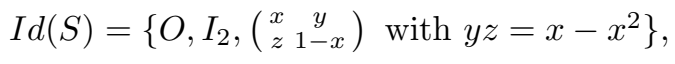

and

$$
P(S)=\left\{O, I_{2},\left(\begin{array}{ll}
1 & 0 \\
0 & 0
\end{array}\right),\left(\begin{array}{ll}
0 & 0 \\
0 & 1
\end{array}\right),\left(\begin{array}{ll}
2 & 1 \\
1 & 2
\end{array}\right),\left(\begin{array}{ll}
2 & 2 \\
2 & 2
\end{array}\right)\right\} .
$$

We next prove that $\operatorname{psr}(S)=1$. Assume on the contrary. Then there exist $A=\left(\begin{array}{ll}a & b \\ c & d\end{array}\right)$ and $A^{\prime}=\left(\begin{array}{ll}a^{\prime} & b^{\prime} \\ c^{\prime} & d^{\prime}\end{array}\right)$ with $A S+A^{\prime} S=S$ but $A+A^{\prime} P$ is not a unit for any $P \in P(S)$. That is,

$$
\operatorname{det}\left(A+A^{\prime} P\right)=0 .
$$

This implies the following system of equations:

$$
\begin{aligned}
& a d-b c=0 \quad \text { (i), } \quad a d^{\prime}-b^{\prime} c=0 \quad \text { (ii), } \\
& a^{\prime} d-b c^{\prime}=0 \quad \text { (iii), } \quad a^{\prime} d^{\prime}-b^{\prime} c^{\prime}=0 \quad \text { (iv), } \\
& a c^{\prime}-a^{\prime} c=b d^{\prime}-b^{\prime} d \quad(\mathrm{v}) \text {. }
\end{aligned}
$$

On the other hand, as isr $(S)=1$, there exists $E \in I d(S) \backslash P(S)$ such that $A+A^{\prime} E \in U(S)$. Then $E$ must be of the form $\left(\begin{array}{cc}x & y \\ z & 1-x\end{array}\right)$ where $y z=x-x^{2}$. By Eqs. (i)-(iv), we obtain

$$
\operatorname{det}\left(A+A^{\prime} E\right)=\left(a c^{\prime}-a^{\prime} c\right) y-\left(b d^{\prime}-b^{\prime} d\right) z .
$$

Next we show that $a c^{\prime}-a^{\prime} c=b d^{\prime}-b^{\prime} d=0$. 
Case 1. $c \neq 0$. Multiplying Eq. (v) by $c$ and by substituting $b^{\prime} c=a d^{\prime}$, we have $\left(a c^{\prime}-a^{\prime} c\right) c=b d^{\prime} c-b^{\prime} d c=(b c-a d) d^{\prime}=0$ by Eq. (i). Thus, $a c^{\prime}-a^{\prime} c=$ $b d^{\prime}-b^{\prime} d=0$.

Case 2. $d \neq 0$. Multiplying Eq. (v) by $d$ and by substituting $a^{\prime} d=b c^{\prime}$, we have $\left(b d^{\prime}-b^{\prime} d\right) d=a c^{\prime} d-a^{\prime} c d=(a d-b c) c^{\prime}=0$ by Eq. (i). So $a c^{\prime}-a^{\prime} c=$ $b d^{\prime}-b^{\prime} d=0$.

Case 3. $c=d=0$. From Eqs. (ii) and (iii), we get $a d^{\prime}=b c^{\prime}=0$. If $b \neq 0$, then $c^{\prime}=0$, it follows that $a c^{\prime}-a^{\prime} c=0$. If $a \neq 0$, then $d^{\prime}=0$, and so $b d^{\prime}-b^{\prime} d=0$. Thus $a c^{\prime}-a^{\prime} c=b d^{\prime}-b^{\prime} d=0$.

Therefore, $\operatorname{det}\left(A+A^{\prime} E\right)=\left(a c^{\prime}-a^{\prime} c\right) y-\left(b d^{\prime}-b^{\prime} d\right) z=0$ for any case, which contradicts $A+A^{\prime} E \in U(S)$. Hence, $\operatorname{psr}(R)=1$.

By Theorem 4.5, we have the following result immediately.

Corollary 4.8. Let $R$ be $a$ *-ring. If $\operatorname{Id}(R)=P(R)$, then the following are equivalent:

(1) $R$ is (strongly) clean.

(2) $R$ is exchange.

(3) $R$ is (strongly) *-clean.

(4) $\operatorname{isr}(R)=1$.

(5) $\operatorname{psr}(R)=1$.

Acknowledgments. The work of the first author was supported by the National Natural Science Foundation of China (No. 11401009; 11326062), Anhui Provincial Natural Science Foundation (No. 1408085QA01) and the Key Natural Science Foundation of Anhui Educational Committee (No. KJ2014A082); and that of the second author by the National Natural Science Foundation of China (No. 11201064) and the Fundamental Research Funds for the Central Universities (No. 2242014R30008).

\section{References}

[1] A. Badawi, On abelian $\pi$-regular rings, Comm. Algebra 25 (1997), no. 4, 1009-1021.

[2] S. K. Berberian, Baer *-Rings, Grundlehren der Mathematischen Wissenschaften, vol. 195, Springer-Verlag, Berlin-Heidelberg-New York, 1972.

[3] K. P. S. Bhaskara Rao, The Theory of Generalized Inverses over Commutative Rings, Taylor \& Francis, London and New York, 2002.

[4] G. Borooah, A. J. Diesl, and T. J. Dorsey, Strongly clean matrix rings over commutative local rings, J. Pure Appl. Algebra 212 (2008), no. 1, 281-296.

[5] V. P. Camillo and H. P. Yu, Exchange rings, units and idempotents, Comm. Algebra 22 (1994), no. 12, 4737-4749.

[6] H. Chen, Rings with many idempotents, Int. J. Math. Math. Sci. 22 (1999), no. 3, $547-558$.

[7] J. Chen and J. Cui, Two questions of L. Vaš on *-clean rings, Bull. Aust. Math. Soc. 88 (2013), no. 3, 499-505.

[8] J. Chen, W. K. Nicholson, and Y. Zhou, Group rings in which every element is uniquely the sum of a unit and an idempotent, J. Algebra 306 (2006), no. 2, 453-460.

[9] J. Chen, X. Yang, and Y. Zhou, When is the $2 \times 2$ matrix ring over a commutative local ring strongly clean? J. Algebra 301 (2006), no. 1, 280-293. 
[10] A. Y. M. Chin and H. V. Chen, On strongly $\pi$-regular group rings, Southeast Asian Bull. Math. 26 (2002), no. 3, 387-390.

[11] C. Li and Y. Zhou, On strongly *-clean rings, J. Algebra Appl. 10 (2011), no. 6, 13631370.

[12] W. K. Nicholson, Lifting idempotents and exchange rings, Trans. Amer. Math. Soc. 229 (1977), 269-278.

[13] Strongly clean rings and Fitting's lemma, Comm. Algebra 27 (1999), no. 8, 3583-3592.

[14] W. K. Nicholson and Y. Zhou, Rings in which elements are uniquely the sum of an idempotent and a unit, Glasg. Math. J. 46 (2004), no. 2, 227-236.

[15] L. Vaš, *-Clean rings: some clean and almost clean Baer *-rings and von Neumann algebras, J. Algebra 324 (2010), no. 12, 3388-3400.

[16] Z. Wang, J. Chen, D. Khurana, and T. Y. Lam, Rings of idempotent stable rang one, Algebr. Represent. Theory 15 (2012), no. 1, 195-200.

JiAN CUI

Department of Mathematics

ANHUi Normal University

Wuhu 241000, P. R. China

E-mail address: cui368@mail.ahnu.edu.cn

ZHOU WANG

Department of Mathematics

Southeast UNIVERSiTy

NAnjing 210096, P. R. China

E-mail address: zhouwang@seu.edu.cn 